\title{
Computer Gaming for Vision Therapy
}

\author{
Tristan Carvelho, Robert S. Allison, Member, IEEE, Elizabeth L. Irving and Christopher Herriot
}

\begin{abstract}
Convergence insufficiency is characterized by an inability to maintain effortless alignment of the two eyes (binocular convergence) while performing near tasks. Conventional rehabilitative vision therapy for the condition is monotonous and dull, leading to low levels of compliance. If the therapy is not performed then improvements in the condition are unlikely. This paper examines the use of computer games as a new delivery paradigm for vision therapy, specifically at how they can be used in the treatment of convergence insufficiency while at home. A game was created and tested in a small scale clinical trial. Results show clinical improvements, as well as high levels of compliance and motivation. Additionally, the game was able to objectively track patient progress and compliance.
\end{abstract}

\section{INTRODUCTION}

\section{A. Physical Rehabilitation}

A large percentage of the population suffers from injuries and neuromuscular disorders which require physical rehabilitation exercises [1]. During this rehabilitation, the patient must perform the same, often monotonous, routine for days, if not months. Expectedly, a large number of patients who require this form of therapy do not comply. In order to keep motivation and morale high, patients must not dread their therapy, and not find it boring or redundant. Therefore, it is important to investigate alternative methods of therapy and to compare their compliance and efficacy to conventional approaches. The use of computer technology has great potential in the physical rehabilitation of these populations.

Computer-assisted physical rehabilitation is a new and rapidly growing area of research and promising advances have been made in areas such as rehabilitation of the upper limbs, the joints of the legs, or gait and posture after stroke or injury [2]-[7]. The majority of applications use haptic interfaces that interact with patients via their sense of touch by applying both assistive and resistive forces. Recently, virtual reality environments have been used to enhance the delivery of this form of therapy [8], [9].

There are number of studies using computer games to aid in the rehabilitation of individuals with both neuromuscular and musculoskeletal disorders with the majority focusing on upper limb rehabilitation (e.g., [10]-[15].). Most of these

Manuscript received April 15, 2008. The authors would like to gratefully acknowledge the support of NSERC and CRC (Canada).

R. S. Allison and T. Carvelho are with the Department of Computer Science and Engineering, York University, Toronto, ON M3J 1P3, Canada (1-416-736-2100; email: \{allison,tristan\}@cse.yorku.ca).

E. L. Irving and C. Herriot are with the School of Optometry, University of Waterloo, Waterloo, ON N2L 3G1, Canada (email: \{elirving, cherriot\}@sciborg.uwaterloo.ca). studies rely on expensive equipment and sophisticated technology to generate suitable forces and to measure the kinematics and dynamics of the limbs. Most studies understandably focus on the devices themselves and concepts such as biofeedback, rather than on the outcomes of clinical trials and factors such as patient motivation and compliance. Vision therapy is a form of physical rehabilitation that does not share the complications related to presenting external forces.

\section{B. Visual Rehabilitation}

Normal binocular vision requires alignment of the two eyes in order to fuse the information provided by each eye into a single image. Many individuals suffer from binocular vision disorders that make this process difficult [16]. Vision therapy or orthoptics is a form of physical therapy. It is a non-surgical approach to treat binocular disorders through the use of "eye exercises". Despite the term "eye exercises" they do not involve strengthening the muscles of the eyes. The primary goal of vision therapy is to improve control of the vergence eye movement system leading to more comfortable and sustainable binocular vision [17]. Differential rotation of the eyes is known as vergence and can either be inward to align the eyes on nearer objects (convergence) or outward to align the eyes on farther objects (divergence).

Binocular alignment is also critical for stereoscopic depth perception. Each of our eyes sees our environment from a slightly different point of view due to their different positions in our head. Differences or disparities between the images received by each eye contain depth information [18].

Depending on the patient and their condition, vision therapy can be performed either at home or under the supervision of an eye care professional. These are referred to as home-based vision therapy (HBVT) and office-based vision therapy (OBVT), respectively. In general, it has been shown that OBVT is more effective and requires a shorter amount of time to work than HBVT [19], [20]. However, OBVT is expensive and requires time away from work, school or other responsibilities and travel to the therapist. The majority of the eye exercises are tedious and monotonous. As such, most patients do not look forward to their therapy and compliance rates are notoriously low [21].

\section{STEREOSCOPIC COMPUTER GAMES FOR VISION THERAPY}

Although the use of computers is a growing area of research in the realm of physical rehabilitation, their use in vision therapy has been limited. Cooper and Citron [22] and Somers et al. [23] presented systems that essentially 
replicated traditional exercises on a computer monitor. Cooper et al. [24] have converted and improved this method so that it could be run at home on a personal computer and it is currently marketed as HTS (Home Vision Therapy System).

We present a new kind of delivery paradigm for homebased vision therapy using video games targeting a common sensory and neuromuscular binocular eye movement disorder known as convergence insufficiency (CI). $\mathrm{CI}$ is characterized by an individual's inability to maintain effortless binocular convergence while performing near visual tasks [16]. Symptoms commonly associated with CI include but are not limited to frontal headaches, blurred vision, double vision, eye strain and reading problems [25], [26]. Typically, treatment for CI relies on exercises that require repeated convergence of the eyes. In our approach, these exercises are integrated into a computer game. Three key interrelated factors to successful vision therapy are motivation, cost, and efficacy. We predict that the use of an interactive medium like video games will make therapy more fun, making patients less likely to be discouraged by their condition and more likely to complete their prescribed therapy with successful outcomes and at modest cost.

\section{A. Game Description}

The game is a modified three-dimensional version of the classic game Pac-Man [27]. In the Stereo Pac-Man game, players navigate Pac-Man through several mazes collecting pellets and power pellets while avoiding four ghosts that emerge from a central cage on the board. Players advance to subsequent levels by collecting all pellets. Collecting power pellets make Pac-Man invincible for a short period of time. If Pac-Man is not "invincible" and he touches a ghost then he will die and all characters will be returned to their starting positions in the maze.

Unlike the original Pac-Man, Stereo Pac-Man makes use of semi-buffered keyboard input making it possible for individuals with slow reaction times to play the game without becoming frustrated with the controls. The player has an unlimited number of lives in Stereo Pac-Man as the length of the training session is set ahead of time and the player can die an arbitrary number of times during this period (i.e. the length of therapy is not based on how good the player is at the game of Pac-Man).

The top portion of the screen consists of a random dot stereogram that contains a top-down view of the current maze. A classical stereogram is composed of a pair of images (one for each eye) that can be viewed using a device known as a stereoscope, which aids in the fusing of the images. In random dot stereograms the images are composed of a random collection of dots. The dot pattern contains "hidden" stereoscopic geometry that can only be seen when the images are properly binocularly aligned. The critical feature of this type of stimuli, that we exploit in our system, is that they allow depth to be perceived from disparity but only when the eyes are properly aligned [28].
The bottom portion of the screen contains a threedimensional stereoscopic representation of the current maze. Power pellets are only visible in the stereogram and regular pellets are only visible in the three dimensional view. There are a total of four randomly placed power pellets per maze, one in each quadrant. If the player presses the spacebar within one second of crossing the power pellet's location (either before or after) they will become invincible, experience an increase in score, and the power pellet will be removed from the stereogram. While invincible, Pac-Man may eat ghosts causing them to return to their starting position in the central cage. The one second window is again to accommodate players with slower reaction times. If the player presses the spacebar when not near a power pellet then they will die, experience a decrease in score, and all characters will be returned to their starting positions in the maze. An image from Stereo Pac-Man can be seen in Figure 1 a.

\section{B. Stereoscopic Viewing and Vergence Demand}

Custom mirror stereoscopes are used to provide each eye with a different image for both the random dot stereogram and the lower three dimensional display. The custom stereoscopes are less costly than current commercial alternatives and include several additional features that make them ideal for home based vision therapy. Sets of mirrors, one set per eye, are directed to opposite halves of the same computer monitor. Therefore, when one looks at the central mirrors each eye receives an image of a different half of the computer screen. As the design is relatively simple, these stereoscopes could be mass-produced using cheaper materials such as plastic. Configuration and setup of the custom mirror stereoscopes is simple and the entire device can be disassembled and reassembled with ease.

The random dot stereograms and the lower display form a stereoscopic stimulus at a simulated distance that is used to provide fusional vergence training to the player. Changing the positions of the monocular stimuli on the computer monitor requires vergence eye movements by the player to fuse them. In this way vergence demand can be varied during the game therapy. A top down view of this setup can be seen in Figure 1b. As the left monocular stimulus is shifted horizontally towards the center of the computer screen and the right monocular stimulus is shifted horizontally towards the center of the computer screen, both the left and right eyes need to turn slightly inwards resulting in an increased convergence angle.

Decreasing separation between the images increases the target (or specified) convergence angle. Similarly, increasing separation between images decreases the convergence angle. The hidden geometry contained in the random dot stereograms is only visible if the eyes are correctly aligned providing a built in check on the player's vergence. If the player correctly identifies a power pellet (by pressing the spacebar when they are in the vicinity of the power pellet) they must be fusing the images correctly and therefore attaining the target level of vergence. At this point 
the separation of the images is reduced to increase the vergence demand of the player. When the player incorrectly identifies a power pellet in the random dot stereogram (i.e. guesses) the separation of the images is increased to lower the vergence demand of the player. This process continues for the duration of the player's therapy. A player cannot advance in the game without using both eyes as the power pellets which must be collected are presented only in the random dot stereograms. Furthermore, the regular pellets are randomly distributed so that approximately half are present in each eye's image, which promotes use of both eyes.

\section{Game Objectives}

1) Motivation: The exercises are tied to the actual gameplay, essentially masking their presence. Success in the game correlates with successful vision therapy. This activity is intrinsically engaging. The game makes use of principles of operant conditioning to motivate the player: positive punishment, positive reinforcement, negative punishment and negative reinforcement [29], [30]. For example, positive reinforcement and negative punishment are employed in relation to the player's score. Whenever the player collects regular pellets, power pellets, and eats ghosts their score increases (positive reinforcement). Whenever the player makes an incorrect guess at the location of a power pellet or is eaten by a ghost their score decreases (negative punishment). More weight was given to the actions that are important to the player's therapy, namely, collecting power pellets and the related act of eating ghosts. Less weight was given to the other actions mentioned as they are purely affect the difficulty of the training (which is set by the clinician). There is no "game over" screen as one of our goals is to have each training session last a specified period of time.

2) Low Cost: The most effective form of vision therapy (office-based) is costly since it requires a trained eye care professional in order to administer the therapy to the patient. The computer game based vision therapy was designed to be performed at home while providing the control and range of exercises typical of office-based therapy. As such it will be much more economical as it only requires the patient to possess or purchase a low cost computer system. In addition, patients will not have to take time away from work or school and can perform their therapy at home at their convenience.

The game is based upon core graphics functionality and requires very little video memory. The three dimensional models created for the game were optimized for speed and all 3D accelerated graphics cards released during or after 1998 support the graphics library and features used by the game. For maximum compatibility the game only relies on functionality from OpenGL version 1.1, which comes preinstalled on Windows 98 and later Windows versions. This covers the vast majority of home PC's sold in the last decade. Additionally, the software can be easily ported to other operating systems.

3) Objective Feedback: Traditional vision therapy requires individuals to visit their eye care practitioner several times in order to determine if there has been any improvement in their condition. Additionally, compliance

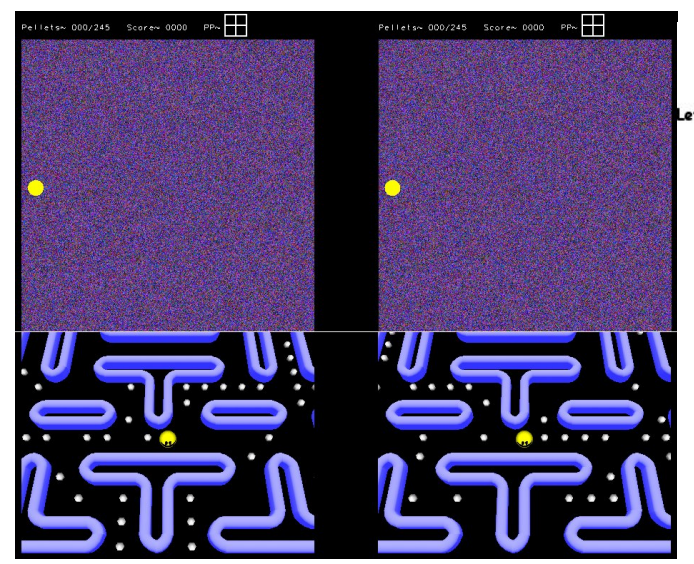

a) A screenshot

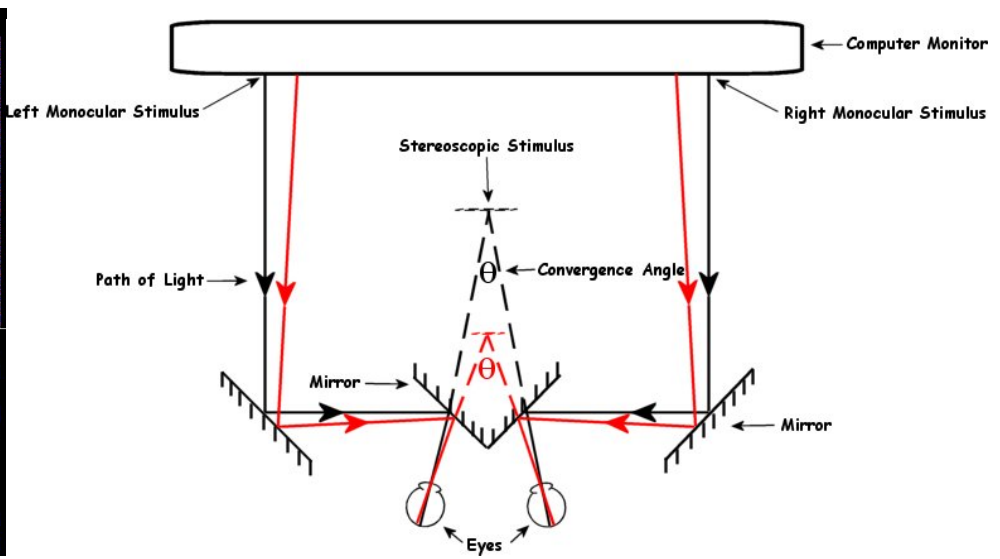

(b) Fusional vergence training using the mirror stereoscope

Fig. 1. Stereo Pacman

related to the game of Pac-Man. The maze game genre was chosen because it appeals to a wide variety of individuals. It appeals to young adults yet does not contain any themes that would be considered unsuitable for children. This genre has a lot of replay value and does not have an "ending", which is important since therapy typically lasts several weeks.

Since individuals have different skills, abilities, and experience, several difficulty levels were implemented. These difficulty levels only affect the game play and do not rates for home-based approaches rely on subjective feedback from the patient. The use of random dot stereograms in the computer game approach makes it possible to objectively track the progress that the patient is making in their therapy. The software is able to verify that the player is actually fusing via their performance in the game.

Furthermore, extensive statistical and logging data are recorded making objective measures of compliance and progress for this new form of home therapy possible. 
Throughout the game, detailed information on convergence levels presented and achieved as well as other parameters related to therapy or game play are recorded and time stamped which makes it possible to determine compliance rates objectively. Hence, the use of a video game automates the tracking of therapy progress and the scheduling of the therapy. In sum, gaming therapy has the potential to provide economical home-based therapy with the benefits of officebased therapy, and improved objective evaluation of compliance and efficacy.

\section{EXPERIMENTAL RESULTS AND EVALUATION}

An experiment was performed to determine if home-based vision therapy incorporated in a computer game would be effective in treating convergence insufficiency and to determine if this new delivery paradigm is motivating and therefore resulting in high levels of compliance. If successful, this study would form the basis for developing a full-scale comparative clinical trial.

\section{A. Method}

The experiment was performed at the School of Optometry, University of Waterloo. All subjects included in the study gave their informed written consent. The research was approved by the Office of Research Ethics at the University of Waterloo and York University. Subjects were given a two-week training protocol to perform in their own home and were assessed at the clinic before the commencement of the training and following each week of training. Two subjects completed an additional 10 training sessions and additional assessments since adjustments to their training protocol were required (see below).

1) Subjects: Seven subjects (ages 11-34) participated in the study. Four of the subjects had a range of symptoms including double vision, eye strain, fatigue, headache, loss of concentration and difficulty with reading during near work. Three were asymptomatic but diagnosed with CI based on the clinical signs discussed below.

2) Vision Training Procedure: Subjects who were eligible for the study were first asked to complete a background questionnaire where they indicated the symptoms that they were currently experiencing. Following this, they were given a demonstration of how to play the game of Stereo Pac-Man. The subjects then took home one of the boxed custom mirror stereoscopes and were asked to play Stereo Pac-Man for 20 minutes a day, 5 days a week, for a 2 week period. In addition, subjects were asked to keep a log of their training times. A set of assembly instructions for the stereoscope, a copy of the Stereo Pac-Man game (on a USB thumb drive), and an explanation of how to configure the game were included within the stereoscope box. To play the game, patients assembled the stereoscope on their home monitor, placed the thumb drive into a free USB slot, and ran the single executable in the root directory of the thumb drive.

3) Clinical Assessment: Subjects were assessed prior to treatment, after the first week of treatment, and at the conclusion of their treatment. We defined CI as the presence of the three most common signs of the condition with or without accompanying symptoms. These signs were a large (or 'receded') near point of convergence (NPC), reduced positive fusional vergence (PFV) at near, and an exophoria at near with thresholds set based on clinical norms. Four clinically relevant metrics were obtained. The NPC break is a measure of how close a target can be brought toward the subjects face before it becomes double (diplopic). PFV is a measure of our ability to converge our eyes in order to maintain clear, single binocular vision. Exophoria is a horizontal outward deviation of the eyes when one is covered and is typically larger at near distances in patients with CI. Exophoria and PFV are usually measured with prisms and the deviations are expressed in units of prism diopters $(\Delta)$, where each prism-diopter corresponds to a one centimeter deviation at a distance of one meter [18]. When the eye looks from a far to a near object it must change focus, or accommodate. This accommodation is accompanied by a change of vergence even when only one eye views. The ratio of the amount of accommodative convergence that accompanies a change in accommodation is the $\mathrm{AC} / \mathrm{A}$ ratio [18]. Although the $\mathrm{AC} / \mathrm{A}$ ratio is not one of our diagnosis criteria it is known to be low in most individuals with CI and was therefore measured at each assessment [31].

4) Subjective Feedback: At the conclusion of treatment subjects were asked to complete a feedback questionnaire regarding their thoughts on this new form of home-based vision therapy. Questions related to possible benefits perceived by the subjects, the ease of use of the system, and the enjoyment or amusement provided by the game were rated on a scale of $1-5$.

\section{B. Results and Analysis}

We discovered that the vergence demand provided by the stereoscope was not sufficient for the first two subjects partway through their training. They were both able to easily reach the maximum convergence angle available so we increased the vergence demand and asked these two subjects to complete ten more training sessions using the modified stereoscope (resulting in 2 more assessments).

Figures $2 \mathrm{a}$ and $2 \mathrm{~b}$ represent the average convergence angle and maximum convergence angle attained by the subjects for each session, respectively. Paired t-tests were performed to determine if the improvements in the average and maximum convergence angles were significant. The mean difference in the average convergence angle from the first to last session was $4.68 \Delta$, with a $95 \%$ confidence interval from -1.16 to $10.53 \Delta$. This improvement was statistically significant, $\mathrm{t}(6)=1.96$, one-tailed $p=4.89 \times 10^{-2}$. There was a significant positive correlation between session number and average convergence angle $(r=0.96, p=9.66 \times$ $\left.10^{-9}\right)$. The mean difference in the maximum convergence angle from the first to last session was $5.01 \Delta$, with a $95 \%$ 
confidence interval from -1.47 to $11.49 \Delta$. This difference was not statistically significant, $\mathrm{t}(6)=1.89$, one-tailed $p=$ $5.38 \times 10^{-2}$. There was a significant positive correlation between session number and maximum convergence angle $(r$ $=0.94, p=1.57 \times 10^{-7}$ ).

Figures $3 \mathrm{a}$ and $3 \mathrm{~b}$ represent the average change in the clinical signs of NPC break and PFV break at near experienced by subjects at each assessment, respectively. As mentioned previously, the first assessment occurred prior to any training and the final assessment at the conclusion of the training. Paired t-tests were performed to determine if the improvements in the NPC break and PFV break at near were significant. The mean difference in the NPC break from the first to last session was $-5.82 \mathrm{~cm}$, with a $95 \%$ confidence interval from -10.69 to $-0.95 \mathrm{~cm}$. This improvement was statistically significant, $\mathrm{t}(6)=2.93$, onetailed $p=1.32 \times 10^{-2}$. The mean difference in the PFV break at near from the first to last session was $12.71 \Delta$ base out (BO), with a 95\% confidence interval from 3.63 to $21.80 \Delta$ BO. This improvement was statistically significant, $\mathrm{t}(6)=3.42$, one-tailed $p=7.05 \times 10^{-3}$. All seven subjects showed an NPC break improvement trend, six of the seven subjects showed a PFV break at near improvement trend, four of the seven subjects showed an AC/A ratio improvement trend, and three of the seven subjects showed a near exophoria improvement trend.

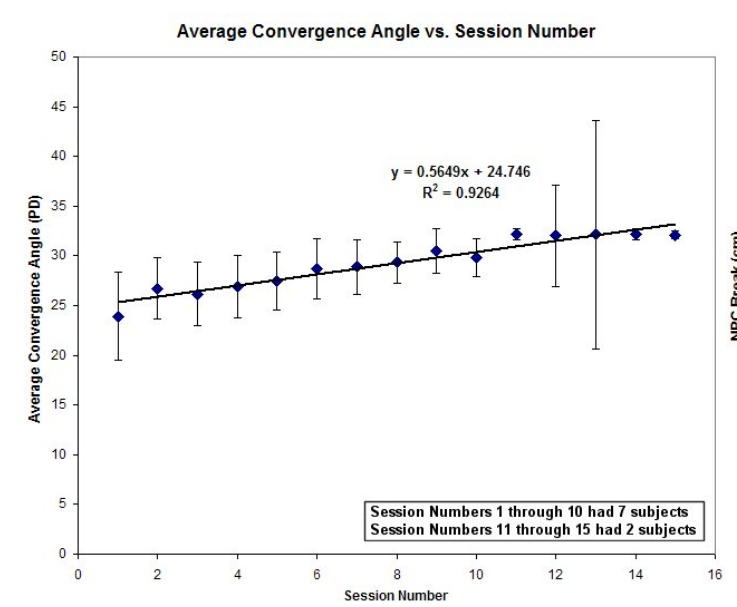

a) Average incorrectly identify a power pellet the vergence demand is decreased. The overall high identification percentage for the power pellets $(87.0 \pm 20.5 \%)$ by all subjects shows that they correctly converged on the target stimuli of the game. Ideally, it should take subjects only a few sessions to become accustomed to the game and reach a near perfect identification percentage. The correct identification percentage for each patient increased over the number of sessions indicating that they were progressing in both the game and their therapy. There was a significant positive correlation between session number and correct power pellet determinations $\left(r=0.92, p=9.62 \times 10^{-7}\right)$ and also with other measures of performance in the game.

Improved performance in the game meant an overall increase in the vergence demand of their training leading to better results. The improvements in the subjects clinical signs increased as well, with larger improvements occurring during later assessments. The fact that the majority of changes were improvements is promising considering the power of the sample.

In terms of compliance, all subjects retained a log of their time spent training. Each subject indicated that exactly 20 minutes were spent training during each of their sessions and the time stamped log files confirmed that this was true on average (20.68 \pm 3.69 minutes). Six of the subjects maintained the training schedule of five days per week,

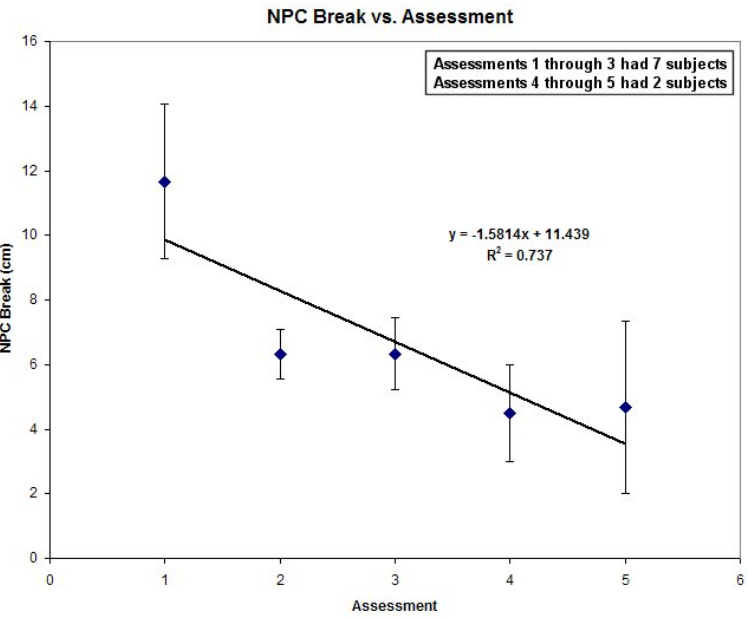

(b) Maximum

Fig. 2. Average and maximum convergence angle per session

At the conclusion of the study, three subjects possessed what is considered a normal NPC break, all seven subjects possessed a normal or better than normal PFV break at near, and three subjects possessed a normal exophoria at near. Additionally, all four of the symptomatic subjects experienced a reduction in eye strain with two of these subjects experiencing a complete elimination of their symptoms. With the exception of two subject's near exophoria, all changes were improvements.

The effectiveness of the therapy is linked to how well subjects perform in the game. When they correctly identify a power pellet the vergence demand is increased. When they while one subject was more inconsistent (at one point, missing upwards of five days). Nevertheless, this subject showed the most improvement.

Analysis of the questionnaire responses indicated that all subjects found the training easy to understand and perform, highly motivating, and amusing. Six of the seven subjects felt that their eye coordination had improved. Four subjects felt less eye strain when looking at near objects and five subjects felt that they benefited from the exercises.

\section{Discussion}

The initial results are promising as they show an 
improvement in the clinical signs used to diagnose CI. Additionally, the AC/A ratio, which was not one of the inclusion criteria, also increased in four of the seven subjects. Four of the seven participants had improved symptoms. The other three were asymptomatic to begin with, explaining their lack of improvement in symptoms. Nonetheless, they did show improvement in all three clinical signs. Compliance is a serious issue with all forms of therapy. All subjects misjudged the amount of time they spent training (both above and below), but the average time they spent training was approximately the required 20 minutes. In future versions of the game, the length of the training could be set programmatically. However, this is no guarantee that players will physically play the game for the entire 20 minutes. It was postulated that the use of a video game would result in improved motivation and compliance. The outcome questionnaire results indicate that this was the case as all seven subjects found the therapy to be motivating and amusing.

Each subject was still making progress in their therapy at the conclusion of their treatment. This leads one to believe that a longer treatment time, allowing for more learning time with the game and how it is played, could have led to better results. This is especially true for the subject with the inconsistent training regimen, who had the longest learning curve with respect to correctly identifying power pellets, resulting in a delay of their training since they attained a

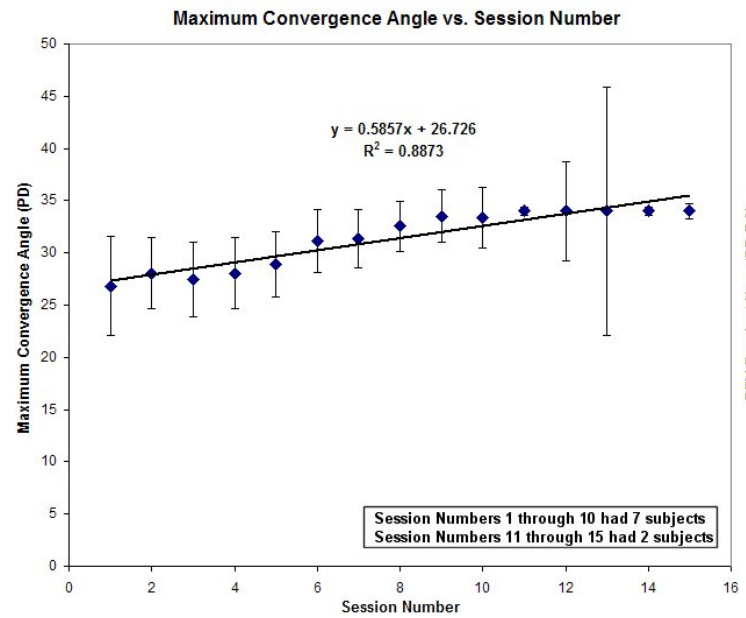

a) $\mathrm{NPC}$ controlled trials.

\section{CONCLUSION}

The system presented is a home-based method of vision therapy. The game has very low system requirements and is capable of running on a wide range of hardware. This means that, in developed countries, the majority of patients will not need to purchase any additional equipment in order to play the game. In addition, custom mirror stereoscopes can be loaned to the patient and are cheaper than current commercial alternatives.

Success in the game relates to successful vision training. Currently, an eye care professional must perform a series of tests on their patient in order to determine how much their condition has improved through training. The game is designed to keep track of how well the patient is doing in their therapy and this data can be used by therapists to adjust their patients training regimen. An online version of the game, or email, could be used to avoid multiple clinic visits.

Computer-game based therapy offers great promise. Successful vision therapy for convergence insufficiency patients reduces the frequency of their headaches, focusing difficulties, eyestrain, and double vision when performing near work or reading. This improves their performance at work and/or school as well as increases their overall quality of life [16].

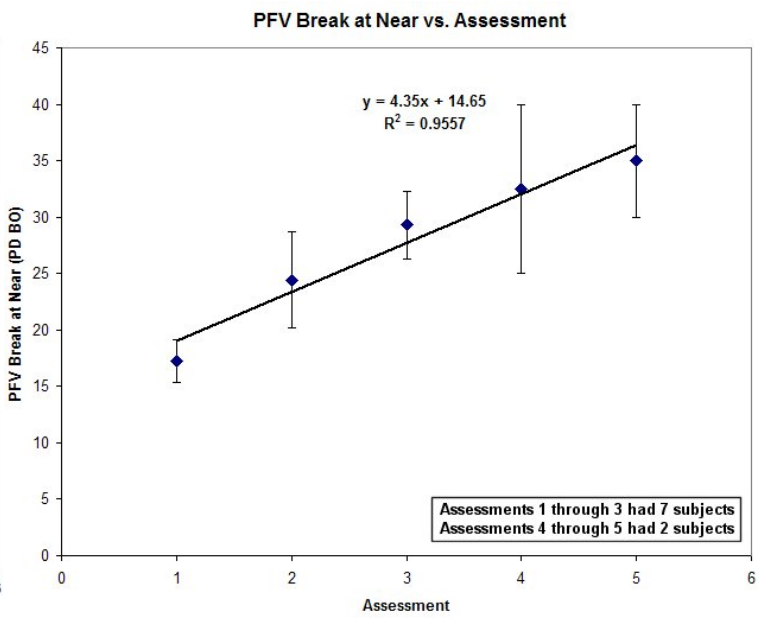

(b) PFV

Fig. 3. NPC and PFV at each assessment

very low vergence demand during approximately their first five sessions. In a retrospective CI home-based vision therapy study by Daum [32], it was indicated that it took 4.3 \pm 4.1 weeks for subjects to show at least some signs of improvement with their condition. Therefore, more treatment time could have resulted in better outcomes. Following the initial pilot study, the goal was to compare computer game vision therapy to pencil pushup therapy, which is the most commonly prescribed home treatment for CI. This has not yet been possible. The experiment is still ongoing and will eventually include conventional therapy

\section{REFERENCES}

[1] American Academy of Orthopaedic Surgeons, "Your orthopaedic connection: Orthopaedic fast facts," http://orthoinfo.aaos.org/topic.cfm?topic=A00130, September 2007.

[2] O. Lambercy, L. Dovat, R. Gassert, E. Burdet, C. Teo, and T. Milner, "A haptic knob for rehabilitation of hand function," IEEE Transactions on Neural Systems and Rehabilitation Engineering, vol. 15, pp. 356-366, 2007.

[3] Q. Li, D. Wang, Z. Du, and L. Sun, "A novel rehabilitation system for upper limbs," in Proceedings of the 2005 IEEE Engineering in Medicine and Biology 27th Annual Conference, September 2005, pp. $6840-6843$. 
[4] S. Dong, K. Lu, J. Sun, and K. Rudolph, "Adaptive force regulation of muscle strengthening rehabilitation device with magnetorheological fluids," IEEE Transactions on Neural Systems and Rehabilitation Engineering, vol. 14, pp. 55-63, 2006.

[5] J. Deutsch, J. Latonio, G. Burdea, and R. Boian, "Post-stroke rehabilitation with the rutgers ankle system: A case study," Presence: Teleoperators and Virtual Environments, vol. 10, pp. 416-430, 2001.

[6] [N. Kim, C. Yoo, and J. Im, "A new rehabilitation training system for postural balance control using virtual reality technology," IEEE Transactions on Rehabilitation Engineering, vol. 7, pp. 482-485, 1999.

[7] R. Ekkelenkamp, J. Veneman, and H. Kooij, "Lopes: selective control of gait functions during the gait rehabilitation of cva patients," in Proceedings of the 2005 IEEE 9th International Conference on Rehabilitation Robotics, July 2005, pp. 361-364.

[8] M. Johnson, B. Ramachandran, R. Paranjape, and J. Kosasih, "Feasibility study of theradrive: a low-cost game-based environment for the delivery of upper arm stroke therapy," in Proceedings of the 28th IEEE Engineering in Medicine and Biology Society Annual International Conference, August 2006, pp. 695-698.

[9] K. McConville and S. Virk, "Motor learning in a virtual environment for vestibular rehabilitation," in Proceedings of the 3rd International IEEE Engineering in Medicine and Biology Conference on Neural Engineering, May 2007, pp. 600-603.

[10] R. Leder, N. Murillo, C. Ibarra, H. Gushiken, G. Anaya, C. Escalona, I. Nunez, A. Alvarez, M. Tyler, and P. Bach-y-Rita, "Computer game motivating rehabilitation with objective measures of improvement in motor function," in 2001 Proceedings of the 23rd Annual Engineering in Medicine and Biology Society International Conference, October 2001, pp. 1388-1390.

[11] M. Elk and B. Driessen, "Active rehabilitation of cva patients," in 2004 IEEE International Conference on Systems, Man and Cybernetics, October 2004, pp. 2449-2453.

[12] A. Betker, T. Szturm, and Z. Moussavi, "Development of an interactive motivating tool for rehabilitation movements," in Proceedings of the 2005 IEEE Engineering in Medicine and Biology 27th Annual Conference, September 2005, pp. 6893-6896.

[13] R. Sanchez, J. Liu, S. Rao, P. Shah, R. Smith, T. Rahman, S. Cramer, J. Bobrow, and D. Reinkensmeyer, "Automating arm movement training following severe stroke: functional exercises with quantitative feedback in a gravity-reduced environment," in IEEE Transactions on Neural Systems and Rehabilitation Engineering, September 2006, pp. 378-389.

[14] J. Azpiroz, F. Barrios, M. Carrillo, R. Carrillo, A. Cerrato, J. Hernandez, R. Leder, A. Rodr'iguez, and P. Salgado, "Game motivated and constraint induced therapy in late stroke with fmri studies pre and post therapy," in Proceedings of the 2005 IEEE Engineering in Medicine and Biology 27th Annual Conference, September 2005, pp. 3695-3698.

[15] A. Heuser, H. Kourtev, S. Winter, D. Fensterheim, G. Burdea, V.
Hentz, and P. Forducey, "Telerehabilitation using the rutgers master II glove following carpal tunnel release surgery: proof-of-concept," in IEEE Transactions on Neural Systems and Rehabilitation Engineering, March 2007, pp. 43-49.

[16] J. Cooper, C. Burns, S. Cotter, K. Daum, J. Griffin, and M. Scheiman, Care of the Patient with Accommodative and Vergence Dysfunction.St. Louis, Missouri: American Optometric Association, 1998.

[17] K. Ciuffreda, "The scientific basis for and efficacy of optometric vision therapy in nonstrabismic accommodative and vergence disorders," Optometry, vol. 73, pp. 735-762, 2002.

[18] I. Howard, Seeing in Depth, chapters 1, 5, 7, 9, 15, 19 and 30. Toronto, Ontario: I. Porteous, 2002.

[19] G. Peachey, "As influenced by the delivery mode," Journal of Behavioral Optometry, vol. 15, pp. 91-97, 2004.

[20] M. Birnbaum, R. Soden, and A. Cohen, "Efficacy of vision therapy for convergence insufficiency in an adult male population," Journal of the American Optometric Association, vol. 70, pp. 225-232, 1999.

[21] M. Gallaway, M. Scheiman, and K. Malhotra, "The effectiveness of pencil pushups treatment for convergence insufficiency: a pilot study," Optometry and Vision Science, vol. 79, pp. 265-267, 2002.

[22] J. Cooper and M. Citron, "Microcomputer produced anaglyphs for evaluation and therapy of binocular anomalies," Journal of the American Optometric Association, vol. 54, pp. 785-788, 1983.

[23] W. Somers, A. Happel, and J. Phillips, "Use of a personal microcomputer for orthoptic therapy," Journal of the American Optometric Association, vol. 55, pp. 262-267, 1984.

[24] J. Cooper, "Diagnosis and treatment of accommodative and vergence anomalies using computerized vision therapy," Practical Optometry, vol. 9, pp. 6-11, 1998.

[25] P. Adler, "Efficacy of treatment for convergence insufficiency using vision therapy," Ophthalmic and Physiological Optics, vol. 22, pp. $565-571,2002$.

[26] K. Daum, "Convergence insufficiency," American Journal of Optometry and Physiological Optics, vol. 61, pp. 16-22, 1984.

[27] Namco, "Pac-man," 1980.

[28] B. Julesz, "Binocular depth perception without familiarity cues," Science, vol. 145, pp. 356-362, 1964.

[29] B. Skinner, The Behavior of Organisms: an Experimental Analysis. New York, New York: D. Appleton Century Crofts, 1938.

[30] J. Cooper and J. Feldman, "Operant conditioning of fusional convergence ranges using random dot stereograms," American Journal of Optometry and Physiological Optics, vol. 57, pp. 205-213, 1980.

[31] K. Daum, "Characteristics of convergence insufficiency," American Journal of Optometry and Physiological Optics, vol. 65, pp. 426-438, 1988.

[32] - "Characteristics of exodeviations: Ii. changes with treatment with orthoptics," American Journal of Optometry and Physiological Optics, vol. 63, pp. 244-251, 1986. 